\title{
Existence results for periodic boundary value problem with a convenction term
}

\author{
Pasquale Candito*, Roberto Livrea*
}

October 31, 2019

Keywords: Periodic BVP, Positive solutions; Coincidence points.

Abstract: By using an abstract coincidence point theorem for sequentially weakly continuous maps the existence of at least one positive solution is obtained for a periodic second order boundary value problem with a reaction term involving the derivative $u^{\prime}$ of the solution $u$; the so called convention term. As consequence of the main result also the existence of at least one positive solution is obtained for a parameter-depending problem.

2010 AMS Subject Classification: 34B15, 34B18

\section{Introduction}

The aim of this paper is to obtain new existence results for the following periodic boundary value problem

$$
\left\{\begin{array}{l}
-u^{\prime \prime}+M(t) u=f\left(t, u, u^{\prime}\right) \\
u(T)-u(0)=u^{\prime}(T)-u^{\prime}(0)=0,
\end{array} \quad \text { in }(0, T)\right.
$$

where $T>0, M:[0, T] \rightarrow \mathbb{R}$ is a continuous and positive function and $f:$ $[0, T] \times \mathbb{R} \times \mathbb{R} \rightarrow \mathbb{R}$ is a continuous function with $f(t, 0,0) \neq 0$, for every $t \in[0, T]$.

As usual, here we say that problem (1.1) has a convention term because the nonlinearity $f$ depends both on the function $u$ and its derivative $u^{\prime}$.

Concerning boundary value problems there is a well consolidated literature where many pioneering results are obtained by several scholars using different tools, as for instance, a priori bounds and topological degree [8, 10, 22]; upper and lower methods $[7,14,24]$ and fixed point theory [1] and [11].

In particular, as pointed out in [25], the application of the fixed point theorem in studying problem (1.1) is strictly connected to the sign properties of the Green's function associated to the linear homogeneous problem, that is $f \equiv 0$.

* The research was supported by the Ministry of Education, University and Research of Italy, Prin 2017 Nonlinear Differential Problems via Variational, Topological and Set-valued Methods, Project No. 2017AYM8XW 
Recently, many authors paid attention to this topic and very interesting results are pointed out in $[2,5,12,13,15,18,20,26,27]$.

Here, for obtaining our main results, we apply a coincidence point theorem for sequentially weakly continuous maps [3], see Theorem 2.1 below, in the variational setting used in [23]. Such approach in spirit is based on an useful version of K. Fan's fixed point theorem [9] contained in [4]. However, we do not use the Green's function to get the solutions of problem (1.1). Moreover, we do not require any asymptotic growth condition on the nonlinearity $f$ at zero and/or at infinity. We just assume condition (3.2) below, together $f(t, 0,0) \neq 0$, for every $t \in[0, T]$ to guarantee the existence of a nontrivial solution which become positive provided that $f(t, 0,0)>0$ for every $t \in[0, T]$.

However, as far as we know, there are few papers dealing with problem (1.1). For example, in [19], applying a coincidence degree theorem and when the nonlinear term is of the form $f(t, x, y)=h(t) g(x, y)$, the existence of at least one positive solution is ensured in terms of the relative behaviors of $\frac{g(x, y)}{|x|+|y|}$ for $|x|+|y|$ near 0 and $+\infty$, where

$(H) h:[0, T] \rightarrow[0,+\infty)$ and $g:[0,+\infty) \times \mathbb{R} \rightarrow[0,+\infty)$ are continuous, $h(t) \not \equiv 0$.

Furthermore, for the readers interested to the applications of periodic BVP in physics and engineering, we again mention [19] and the references therein.

On the other hand, it seems that much more attention is paid to problems without convention terms and depending from a positive parameter $\lambda$. An example is the following

$$
\left\{\begin{array}{l}
-u^{\prime \prime}+M(t) u=\lambda g(t, u) \\
u(T)-u(0)=u^{\prime}(T)-u^{\prime}(0)=0,
\end{array} \quad \text { in }(0, T)\right.
$$

where $T>0, M:[0, T] \rightarrow \mathbb{R}$ is a continuous and positive function and $g:$ $[0, T] \times \mathbb{R} \rightarrow \mathbb{R}$ is a continuous function.

In this case, many existence, non-existence and multiplicity results have been obtained, for instance, in $[12,13,16,17,20,21,27]$, requiring suitable asymptotic behaviors of the "slope" $f(t, u) / u$ of $f$ at zero and at infinity.

Finally, for the sake of completeness, we wish to stress that in [3] and [6] a similar approach to those proposed in the present note has been adopted for the study of a Dirichlet and a Neumann boundary value problem respectively.

\section{Preliminaries}

We recall that the weak derivative of a function $u \in L^{1}([0, T])$ is a function $u^{\prime} \in L^{1}([0, T])$ such that

$$
\int_{0}^{T} u(t) \varphi^{\prime}(t) d t=-\int_{0}^{T} u^{\prime}(t) \varphi(t) d t
$$

for every $\varphi \in C_{T}^{\infty}$, where $C_{T}^{\infty}$ is the space of indefinitely differentiable $T$-periodic functions (see [23]). 
Let us denote by $H_{T}$ the Sobolev space of functions $u \in L^{2}([0, T])$ having a weak derivative $u^{\prime} \in L^{2}([0, T])$, while

$$
H_{T}^{2}=\left\{u \in H_{T}: u^{\prime} \in H_{T}\right\} .
$$

According to ([23, pp. 6-7]), for every $u \in H_{T}^{2}$ one has that

$$
\int_{0}^{T} u^{\prime}(t) d t=\int_{0}^{T} u^{\prime \prime}(t) d t=0,
$$

hence the periodic conditions $u(T)-u(0)=u^{\prime}(T)-u^{\prime}(0)=0$ hold. Moreover, if we endow $H_{T}^{2}$ with the norm

$$
\|u\|=\|u\|_{2}+\left\|u^{\prime}\right\|_{2}+\left\|u^{\prime \prime}\right\|_{2}
$$

for every $u \in H_{T}^{2}$ and on $C^{1}([0, T])$ we consider the norm

$$
\|u\|_{C^{1}}=\max \left\{\|u\|_{\infty},\left\|u^{\prime}\right\|_{\infty}\right\},
$$

$H_{T}^{2}$ is compactly embedded in $C^{1}([0, T])$, see $[23$, Proposition 1.2]. In particular, if $u \in H_{T}^{2}$ observe that

$$
\begin{aligned}
|u(t)| & =\frac{1}{T}\left|\int_{0}^{T} u(s)+\int_{0}^{T}\left(\int_{s}^{t} u^{\prime}(x) d x\right) d s\right| \\
& \leq \frac{1}{T}\|u\|_{1}+\left\|u^{\prime}\right\|_{1} \leq T^{-1 / 2}\|u\|_{2}+T^{1 / 2}\left\|u^{\prime}\right\|_{2} \\
& \leq \max \left\{T^{-1 / 2}, T^{1 / 2}\right\}\|u\|
\end{aligned}
$$

for every $t \in[0, T]$. Thus, if we put

$$
c_{T}=\max \left\{T^{-1 / 2}, T^{1 / 2}\right\}
$$

one can conclude that

$$
\|u\|_{\infty} \leq c_{T}\|u\|
$$

Similarly one can obtains

$$
\left\|u^{\prime}\right\|_{\infty} \leq c_{T}\|u\|
$$

namely

$$
\|u\|_{C^{1}} \leq c_{T}\|u\| .
$$

Incidentally, observe that if $0<T \leq 1$ then $c_{T}=T^{-1 / 2}$ and one can realize the equality in (2.4) choosing $u$ constant. Namely, if $0<T<1$ the constant introduced in (2.1) is the best one of the embedding. Some sharp estimates for the norms of functions in $H_{T}$ can be found in [23, Proposition 1.3].

A direct computation based on (2.4) shows that for every $r>0$

$$
B_{r}=\left\{u \in H_{T}^{2}:\|u\| \leq r\right\} \subseteq\left\{u \in C^{1}([0,1]):\|u\|_{C^{1}} \leq c_{T} r\right\} .
$$

The following coincidence point theorem represents the key tool for the proof of our main results. 
Theorem 2.1. Let $X, Y$ be real Banach spaces, let $K$ be a weakly compact, convex subset of $X$, and let $F, G$ be sequentially weakly continuous functions from $K$ into $Y$, that is, if $x_{n} \rightarrow x$ in $K$ then $F\left(x_{n}\right) \rightarrow F(x)$ and $G\left(x_{n}\right) \rightarrow G(x)$ in $Y$. Assume that $F^{-1}(y)$ is a nonempty convex set for all $y \in G(K)$. Then there exists $x_{0} \in K$ such that $F\left(x_{0}\right)=G\left(x_{0}\right)$.

\section{Main results}

Here is the first existence result for the considered periodic problem.

Theorem 3.1. Let $f:[0, T] \times \mathbb{R} \times \mathbb{R} \rightarrow \mathbb{R}$ be a continuous function. Put

$$
\tau=\frac{\mu}{c_{T} \sqrt{T}\left[1+(T+1)\left(\|M\|_{\infty}+\mu\right)\right]}
$$

with $\mu=\min _{t \in[0, T]} M(t)$, and assume that there exists $r>0$ such that

$$
\max _{(t, x, y) \in[0, T] \times[-r, r] \times[-r, r]}|f(t, x, y)| \leq \tau \cdot r .
$$

Then, problem (1.1) admits at least one classical solution $\tilde{u}$ such that

$$
\left(\tilde{u}(t), \tilde{u}^{\prime}(t), \tilde{u}^{\prime \prime}(t)\right) \in[-r, r] \times[-r, r] \times\left[-\left(\|M\|_{\infty}+\tau\right) r,\left(\|M\|_{\infty}+\tau\right) r\right] .
$$

Proof. We will apply Theorem 2.1 with $X=H_{T}^{2}, Y=X^{*}, K=B_{\rho}$, being $\rho=\frac{r}{c_{T}}$, and $F, G: X \rightarrow X^{*}$ the functions defined as follows

$$
\begin{gathered}
F(u)(v)=\int_{0}^{T}\left(u^{\prime}(t) v^{\prime}(t)+M(t) u(t) v(t)\right) d t \\
G(u)(v)=\int_{0}^{T} f\left(t, u(t), u^{\prime}(t)\right) d t
\end{gathered}
$$

for every $u, v \in X$. Indeed, $K$ is weakly compact in view of the reflexivity of $X$, while the compactness of the embedding of $X$ into $C^{1}([0, T])$ assures that both $F$ and $G$ are sequentially weakly continuous functions from $X$ to $X^{*}$. We claim that

$$
G(K) \subseteq F(K)
$$

Fix $w^{*} \in G(K)$ and let $w \in K$ be such that $G(w)=w^{*}$. Put

$$
g(t)=f\left(t, w(t), w^{\prime}(t)\right)
$$

for all $t \in[0, T]$ and observe that $g \in C^{0}([0, T])$. Hence, applying the MintyBrowder theorem (or the Lax-Milgram theorem) in the space $H_{T}$, the following problem

$$
\begin{cases}-u^{\prime \prime}+M(t) u=g(t) & \text { in }(0, T) \\ u(T)-u(0)=u(T)-u(0)=0 & \end{cases}
$$


admits a unique weak solution $u_{w} \in H_{T}$ and, in particular, thanks to the classical regularity theory, one has that $u_{w} \in C^{2}([0, T])$ and it is a classical solution.

If we localize $u_{w} \in H_{T}^{2}$ and prove that

$$
u_{w} \in B_{\rho}
$$

we can conclude that $(3.3)$ holds, since $F\left(u_{w}\right)=G(w)=w^{*}$.

To this end, we first point out that

$$
\begin{gathered}
\left\|u_{w}\right\|_{\infty} \leq \frac{\|g\|_{\infty}}{\mu} \\
\left\|u_{w}^{\prime}\right\|_{\infty} \leq T\left(\frac{\|M\|_{\infty}}{\mu}+1\right)\|g\|_{\infty},
\end{gathered}
$$

and

$$
\left\|u_{w}^{\prime \prime}\right\|_{\infty} \leq\left(\frac{\|M\|_{\infty}}{\mu}+1\right)\|g\|_{\infty} .
$$

Indeed, fix $k=\frac{\|g\|_{\infty}}{\mu}$ and put $\varphi(t)=\left(u_{w}-k\right)^{+}$. Obviously $\varphi \in H_{T}$ and $\varphi^{\prime}=u_{w}^{\prime} \cdot \chi_{\left\{u_{w} \geq k\right\}}$. Hence, from (3.4) one has

$$
\int_{0}^{T}\left(u_{w}^{\prime} \varphi^{\prime}+M(t) u_{w} \varphi\right) d t=\int_{0}^{T} g \varphi d t
$$

that is

$$
\begin{aligned}
0 & \leq \int_{0}^{T} M(t)\left(u_{w}-k\right)\left(u_{w}-k\right)^{+} d t \\
& \leq \int_{0}^{T}\left(\left(u_{w}^{\prime}\right)^{2} \chi_{\left\{u_{w} \geq k\right\}}+M(t)\left(u_{w}-k\right)\left(u_{w}-k\right)^{+}\right) d t \\
& =\int_{0}^{T}(g-M(t) k)\left(u_{w}-k\right)^{+} d t \leq 0,
\end{aligned}
$$

and this implies that $\left(u_{w}-k\right)\left(u_{w}-k\right)^{+} \equiv 0$, namely

$$
u_{w}(t) \leq k
$$

for every $t \in[0, T]$. Arguing in a similar way, one has that

$$
-k \leq u_{w}(t)
$$

for every $t \in[0, T]$. Clearly (3.9) and (3.10) lead to (3.6).

Moreover, since $u_{w}(0)=u_{w}(T)$, there exists $t_{0} \in(0, T)$ such that $u_{w}^{\prime}\left(t_{0}\right)=0$ 
and, in view of (3.6), for every $t \in[0, T]$ one has

$$
\begin{aligned}
\left|u_{w}^{\prime}(t)\right| & =\left|\int_{t_{0}}^{t} u_{w}^{\prime \prime}(s) d s\right| \\
& =\left|\int_{t_{0}}^{t}\left(M(s) u_{w}(s)-g(s)\right) d s\right| \\
& \leq T\left(\|M\|_{\infty}\left\|u_{w}\right\|_{\infty}+\|g\|_{\infty}\right) \\
& \leq T\left(\frac{\|M\|_{\infty}}{\mu}+1\right)\|g\|_{\infty},
\end{aligned}
$$

namely (3.7) holds.

Exploiting again that $u_{v}$ is a classical solution of problem (3.4), from (3.6) one derives

$$
\left\|u_{w}^{\prime \prime}\right\|_{\infty} \leq\left(\frac{\|M\|_{\infty}}{\mu}+1\right)\|g\|_{\infty}
$$

and (3.8) is verified.

Now observe that from (2.5) it follows that $\|w\|_{C^{1}} \leq r$, hence, in view of assumption (3.2), $\|g\|_{\infty} \leq \tau \cdot r$. Putting together (3.6)-(3.8) and this last estimate, one has

$$
\left\|u_{w}\right\|_{2}+\left\|u_{w}^{\prime}\right\|_{2}+\left\|u_{w}^{\prime \prime}\right\|_{2} \leq \tau \frac{\sqrt{T}}{\mu}\left[1+(T+1)\left(\|M\|_{\infty}+\mu\right)\right] r=\frac{r}{c_{T}}=\rho,
$$

namely (3.5) holds and (3.3) is verified.

It is simple to verify that $F$ is injective, hence $F^{-1}\left(w^{*}\right)=\left\{u_{w}\right\}$ for every $w^{*} \in G(K)$ and all the assumptions of Theorem 2.1 are satisfied. Thus, there exists $\tilde{u} \in K$ such that

$$
F(\tilde{u})(v)=G(\tilde{u})(v)
$$

for every $v \in H_{T}^{2}$. But $C_{T}^{\infty} \subset H_{T}^{2}$ implies that $\tilde{u}^{\prime} \in H_{T}$, being $M(t) \tilde{u}-f\left(t, \tilde{u}, \tilde{u}^{\prime}\right)$ its weak derivative. The regularity theory assures that $\tilde{u} \in C^{2}([0, T])$ and it is a classical solution of (1.1). The proof is complete since $\|\tilde{u}\|_{\infty}$, and $\left\|\tilde{u}^{\prime}\right\|_{\infty}$ can be estimated recalling (2.5), while $\left\|\tilde{u}^{\prime \prime}\right\|_{\infty}$ can be estimated exploiting the fact that $\tilde{u}$ solves (1.1).

As a consequence of the previous result, we can state the main constant sign periodic solution theorem.

Theorem 3.2. Let $f:[0, T] \times \mathbb{R} \times \mathbb{R} \rightarrow \mathbb{R}$ be a continuous function such that $f(t, 0,0)>0$ for every $t \in[0, T]$. Let $\tau>0$ as defined in (3.1) and assume that

$$
\max _{(t, x, y) \in[0, T] \times[0, r] \times[-r, r]}|f(t, x, y)| \leq \tau \cdot r .
$$

Then, problem (1.1) admits at least one positive classical solution $\tilde{u}$ such that such that

$$
\left(\tilde{u}(t), \tilde{u}^{\prime}(t), \tilde{u}^{\prime \prime}(t)\right) \in(0, r] \times(0, r] \times\left[-\left(\|M\|_{\infty}+\tau\right) r,\left(\|M\|_{\infty}+\tau\right) r\right] .
$$


Proof. We make use of some truncation arguments. Let $\hat{f}:[0, T] \times \mathbb{R} \times \mathbb{R} \rightarrow \mathbb{R}$ be the function defined by

$$
\hat{f}(t, x, y)= \begin{cases}f(t, x, y) & \text { if } x \geq 0 \\ f(t, 0, y) & \text { if } x<0\end{cases}
$$

If we consider the following auxiliary periodic problem

$$
\left\{\begin{array}{l}
-u^{\prime \prime}+M(t) u=\hat{f}\left(t, u, u^{\prime}\right) \quad \text { in }[0, T] \\
u(T)-u(0)=u^{\prime}(T)-u(0)=0,
\end{array}\right.
$$

it is evident that the non negative solutions of (3.13) are also constant sign solutions of problem (1.1). At this point, we can observe that, thanks to (3.11) and (3.12), $\hat{f}$ satisfies all the assumptions of Theorem 3.1. Hence, problem (3.13) admits at least one classical solution $\tilde{u} \in C^{2}([0, T])$. Finally, the proof is complete if we verify that

$$
\min _{t \in[0, T]} \tilde{u}(t)>0
$$

Suppose (3.14) false, namely, there exists $t^{*} \in[0, T]$ such that

$$
\tilde{u}\left(t^{*}\right)=\min _{t \in[0, T]} \tilde{u}(t) \leq 0 .
$$

Thus, we have that

$$
\tilde{u}^{\prime}\left(t^{*}\right)=0, \quad \tilde{u}^{\prime \prime}\left(t^{*}\right) \geq 0 .
$$

Indeed, if $t^{*} \in(0, T)$ then (3.15) is obvious. Otherwise, suppose that $t^{*}=0$ (the other case $t^{*}=T$ is analogous). Since 0 is a minimizer of $\tilde{u}$ one has that $\tilde{u}^{\prime}(0) \geq 0$, but the periodic boundary conditions lead to $\tilde{u}^{\prime}(0)=0$. Otherwise, if $\tilde{u}^{\prime}(0)>0$ one has $\tilde{u}^{\prime}(T)>0$ and for $t$ close to $T$ one achieves the contradiction $\tilde{u}(t)<\tilde{u}(T)=\tilde{u}(0)=\min _{[0, T]} \tilde{u}$.

Moreover, if it was $\tilde{u}^{\prime \prime}(0)<0$, since $\tilde{u} \in C^{2}([0, T])$, one could find a suitable $\delta>0$ such that $\tilde{u}^{\prime}(t)<0$ for all $t \in(0, \delta)$, in contradiction with the fact that $t^{*}=0$ is a minimizer.

At this point, exploiting (3.15) one is lead to the evident contradiction

$$
0 \geq-\tilde{u}^{\prime \prime}\left(t^{*}\right)+M\left(t^{*}\right) \tilde{u}\left(t^{*}\right)=\hat{f}\left(t^{*}, \tilde{u}\left(t^{*}\right), \tilde{u}^{\prime}\left(t^{*}\right)\right)=f\left(t^{*}, 0,0\right)>0 .
$$

In conclusion, (3.14) holds and the proof is completed.

Remark 3.3. The existence of a negative classical solution can be similarly proved if one assumes that $f(t, 0,0)<0$ for every $t \in[0, T]$, in place of $f(t, 0,0)>0$.

Corollary 3.4. Let $T>0, M:[0, T] \rightarrow \mathbb{R}$ a continuous and positive function and $g:[0, T] \times \mathbb{R} \rightarrow \mathbb{R}$ a continuous function. Then, there exists $\lambda^{*}>0$ such that, for each $\lambda \in]-\lambda^{*}, \lambda^{*}[$, problem (1.2) admits at least one classical solution. 
Proof. Let $\tau$ as given in (3.1) and put

$$
\lambda^{*}=\tau \sup _{r>0} \frac{r}{\max _{[0, T] \times[-r, r]}|g(t, x)|} .
$$

Therefore, fixed $\lambda$ such that $|\lambda|<\lambda^{*}$, it is clear that there exists $r>0$ such that

$$
\max _{(t, x) \in[0, T] \times[-r, r]}|\lambda g(t, x)|<\tau r .
$$

In few words, the function $\lambda g$ fulfils condition (3.2) of Theorem 3.1 and our conclusion follows.

Example 3.5. The following problem

$$
\left\{\begin{array}{l}
-u^{\prime \prime}+\frac{u}{2}=\frac{2+\sin (t)}{40 \pi^{2}}\left(1-u^{3}\right)\left(1-u^{4}\right) \\
u(2 \pi)-u(0)=u^{\prime}(2 \pi)-u^{\prime}(0)=0
\end{array} \quad \text { in }[0,2 \pi]\right.
$$

admits at least one positive and non constant solution.

Indeed, we can apply Theorem 3.2 if we consider $r=1, M(t) \equiv 1 / 2$ and put

$$
f(t, x, y)=\frac{2+\sin (t)}{40 \pi^{2}}\left(1-x^{3}\right)\left(1-y^{4}\right)
$$

for every $(t, x, y) \in[0,1] \times \mathbb{R} \times \mathbb{R}$, simple computation shows that

$$
\begin{aligned}
\max _{[0,1] \times[0,1] \times[-1,1]}|f(t, x, y)| & =\max _{[0,1] \times[0,1] \times[-1,1]} \frac{2+\sin (t)}{40 \pi^{2}}\left(1-x^{3}\right)\left(1-y^{4}\right) \\
& =\frac{3}{40 \pi^{2}},
\end{aligned}
$$

namely (3.11) is satisfied, being $\tau=\frac{1}{8 \pi(1+\pi)}$. Hence, (3.16) has at least one positive classical solution $u_{0}$ such that $\left(u_{0}(t), u_{0}^{\prime}(t), u_{0}^{\prime \prime}(t)\right) \in(0,1] \times(0,1] \times$ $\left[-\frac{1}{2}-\frac{1}{8 \pi(1+\pi)}, \frac{1}{2}+\frac{1}{8 \pi(1+\pi)}\right]$ for every $t \in[0,1]$. Finally, it is easy to verify that (3.16) does not admits constant solutions..

\section{References}

[1] H. Amann, Fixed point equations and nonlinear eigenvalue problems in ordered Banach spaces. SIAM Rev. 18(4), (1976), 620-709.

[2] F.M. Atici, G.Sh. Guseinov, On the existence of positive solutions for nonlinear differential equations with periodic boundary conditions, J. Comput. Appl. Math. 132 (2001), 341-356.

[3] G. Bonanno, P. Candito, D. Motreanu, A coincidence point theorem for sequentially continuous mappings, J. Math. Anal. Appl. 435 (2016), no. 1, 606-615. 
[4] G. Bonanno, S.A. Marano, Positive solutions of elliptic equations with discontinuous nonlinearities, Topol. Methods Nonlinear Anal., 8 (1996), 263-273.

[5] A. Cabada, J. Cid, Existence and multiplicity of solutions for a periodic Hill's equation with parametric dependence and singularities, Abstr. Appl. Anal. 2011, Art. ID 545264, 19 pp.

[6] P. Candito, R. Livrea, An existence result for a Neumann problem, Dyn. Contin. Discrete Impuls. Syst. Ser. A Math. Anal. 22 (2015), 481-488.

[7] C. De Coster, P. Habets, Upper and lower solutions in the theory of ODE boundary value problems: classical and recent results, in F. Zanolin (Ed.), Nonlinear analysis and boundary value problems for ordinary differential equations (Udine), 178, CISM Courses and Lect., 371, Springer, Vienna, 1996.

[8] P. Drábek, Landesman-Lazer condition for nonlinear problems with jumping nonlinearities, J. Differential Equations 85 (1990), 186-199.

[9] K. Fan, Fixed-point and minimax theorems in locally convex topological linear spaces, Proc. Natl. Acad. Sci. USA, 38 (1952), 121-126.

[10] R. E. Gaines, J. Mawhin, Coincidence degree, and nonlinear differential equations. In: LNM, vol. 568. Springer, Berlin (1977).

[11] D. Guo, V. Lakshmikantham, Nonlinear Problems in Abstract Cones, Academic Press, Orlando, FL, 1988.

[12] J. Graef, L. Kong, H. Wang, A periodic boundary value problem with vanishing Green's function, Appl. Math. Lett. 21 (2008), no. 2, 176-180.

[13] J. Graef, L. Kong, H. Wang, Existence, multiplicity, and dependence on a parameter for a periodic boundary value problem, Differential Equations 245, (2008), 1185-1197.

[14] P. Habets, L. Sanchez, Periodic solutions of some Liénard equations with singularities, Proc. Amer. Math. Soc. 109 (1990), no. 4, 1035-1044.

[15] D. D. Hai, Existence of positive solutions for periodic boundary value problem with sign-changing Greens function, Positivity 22 (2018), no. 5, 12691279 .

[16] X. Hao, L. Liu, Y. Wu, Existence and multiplicity results for nonlinear periodic boundary value problems, Nonlinear Anal. 72, (2010), 3635-3642.

[17] Z. He, R. Ma, M. Xu, Positive solutions for a class of semipositone periodic boundary value problems via bifurcation theory, Electron. J. Qual. Theory Differ. Equ. 2019, Paper No. 29, 15 pp.

[18] D. Jiang, J. Chu, D. ORegan, R. Agarwal, Multiple positive solutions to superlinear periodic boundary value problems with repulsive singular forces, J. Math. Anal. Appl. 286 (2003), 563-576. 
[19] J. Liu, H. Feng, Positive solutions of periodic boundary value problems for second-order differential equations with the nonlinearity dependent on the derivative, J. Appl. Math. Comput. 49 (2015), no. 1-2, 343-355.

[20] R. Ma, Nonlinear periodic boundary value problems with sign-changing Greens function, Nonlinear Anal. 74 (2011), no. 5, 1714-1720.

[21] R. Ma, J. Xu, X. Han, Global structure of positive solutions for superlinear second-order periodic boundary value problems, Appl. Math. Comput. 218 (2012), 5982-5988.

[22] J. Mawhin, Topological degree and boundary value problems for nonlinear differential equations M. Furi, P. Zecca (Eds.), Topological methods for ordinary differential equations, Lecture Notes in Mathematics, Vol. 1537, Springer, New York/Berlin (1993), pp. 74-142.

[23] J. Mawhin, M. Willem, Critical Point Theory and Hamiltonian Systems, Springer-Verlag, New York, 1989.

[24] I. Rachůnková, M. Tvrdý, Nonlinear systems of differential inequalities and solvability of certain boundary value problems, J. Inequal. Appl. 6 (2001), no. 2, 199-226.

[25] P. J. Torres, Existence of one-signed periodic solutions of some second-order differential equations via a Krasnoselskii fixed point theorem, J. Differential Equations 190 (2003), no. 2, 643-662.

[26] H. Wang, On the number of positive solutions of nonlinear systems, J. Math. Anal. Appl. 281 (2003), 287-306.

[27] J. R. L. Webb, Boundary value problems with vanishing Greens function, Commun. Appl. Anal. 13 (2009), no. 4, 587-595. 